\title{
Outcome Measure of Pain in Patients with Lumbar Disc Herniation: Validation Study of the Iranian version of Pain Sensitivity Questionnaire
}

\author{
Parisa Azimi ${ }^{1}$, Shirzad Azhari ${ }^{1}$, Sohrab Shahzadi ${ }^{1}$, Hossain Nayeb Aghaei ${ }^{1}$, \\ Hassan Reza Mohammadi ${ }^{1}$, Ali Montazeri ${ }^{2}$ \\ ${ }^{1}$ Functional Neurosurgery Research Center, Shahid Beheshti University of Medical Sciences, Tehran, Iran \\ ${ }^{2}$ Mental Health Research Group, Health Metrics Research Centre, Iranian Institute for Health Sciences Research, ACECR, Tehran, Iran
}

\begin{abstract}
Study Design: Cross-sectional.
Purpose: To translate and culturally adapt an Iranian version of the Pain Sensitivity Questionnaire (PSQ) in Iran.

Overview of Literature: Instruments measuring patient reported outcomes should satisfy certain psychometric properties.

Methods: The PSO was translated following cross-cultural adaptation guidelines. A total of 101 patients with lumbar disc herniation (LDH), and 39 healthy cases were included in the study. All participants completed the PSO and the Pain Catastrophizing Scale (PCS). The internal consistency, test-retest reliability, known group comparison, criterion validity and item-scale correlations were assessed. Results: The mean age of participants was 51.7 years. Reliability, validity and correlation of PSQ and PCS showed satisfactory results. Cronbach's alpha coefficients were 0.81 for PSQ-total, 0.82 for PSQ-minor, and 0.82 for PSQ-moderate. The intraclass correlation coefficients value was $0.84(0.616-0.932)$ indicating an excellent test-retest reliability. The instrument discriminated well between sub-groups of patients who differed in a standard predictive measure of LDH surgery (the Finneson-Cooper score). Total PSO were also significantly correlated with the total scores of the PCS, lending support to its good convergent validity. Additionally, the correlation of each item with its hypothesized domain on the PSQ indicated acceptable results, suggesting that the items had a substantial relationship with their own domains.

Conclusions: The adapted Iranian PSQ is a valid and reliable questionnaire for the assessment of pain in patients with LDH.
\end{abstract}

Keywords: Iran; Pain Sensitivity Questionnaire; Validity; Lumbar disc herniation

\section{Introduction}

Lumbar disc herniation (LDH) is a displacement of disc material (annulus fibrosis or nucleus pulposus) beyond the intervertebral disc space. LDH typically presents with lower back pain that radiates down one leg, and is often accompanied by numbness or tingling in the foot [1].
Pain is a critical landmark in patients with spinal disorder that requires immediate attention from spine specialists [2]. There are large individual differences in pain perception and pain responsiveness. Pain sensitivity is a potentially useful construct for understanding individual variability in the experience of pain. The Pain Sensitivity Questionnaire (PSQ) is predictive of pain-related

\footnotetext{
Received Sep 9, 2015; Revised Sep 30, 2015; Accepted Oct 1, 2015

Corresponding author: Parisa Azimi

Department of Neurosurgery, Shohada Tajrish Hospital, Functional Neurosurgery Research Center of Shohada Tajrish Hospital, Shahid Beheshti University of Medical Sciences, Tehran, Iran

Tel: +98-212-274-9204, Fax: +98-218-826-5188, E-mail: parisa.azimi@gmail.com
} 
responses to experimental stimuli. It has been developed as a simple and economical alternative to more timeconsuming experimental testing methods that involve expensive equipment. This questionnaire has been proven to be a valid assessment tool for the evaluation of pain sensitivity in both healthy individuals and patients with chronic pain [3]. It has been validated as an outcome measure in chronic pain and degenerative spinal disease $[4,5]$. In addition, the PSQ used to predict surgical outcomes for lumbar spinal stenosis [6].

The aim of this study was to translate the PSQ into Persian (Iranian language), validate and use the questionnaire in studies of pain sensitivity in patients with LDH in Iran. Currently there is no such questionnaire available in Iran.

\section{Materials and Methods}

\section{Questionnaire}

Ruscheweyh et al. developed the PSQ, which assesses general pain sensitivity by self-rating without using extensive and painful experimental stimulation. This questionnaire contains a series of questions in which one should imagine himself in certain situations. Then one should decide if these situations would be painful and, if yes, how painful they would be. It consists of 17 questions, each describing a daily life situation on a numeric rating scale ranging from 0 (not painful at all) to 10 (worst pain imaginable). Of the 17 situations, most healthy subjects could rate 14 items as painful. These painful situations involve a range of painful stimuli including hot, cold, sharp, and blunt stimulation applied to different body parts including the head and upper and lower extremities. However, three of the 17 situations are normally not considering as painful by healthy subjects. These items are not integrating in the final score. Patients require approximately 15 minutes to complete the PSQ with the assistance of a clinical researcher. The PSQ-total score could be calculated as the average rating of items $1,2,3,4,6,7,8,10,11,12,14,15$, 16 , and 17 (all but the three non-painful items). The PSQ also has a minor score (PSQ-minor) and a moderate score (PSQ-moderate). PSQ-minor is calculated as the average rating of seven items $(3,6,7,10,11,12$, and 14). PSQmoderate score is calculated as the average rating of seven items $(1,2,3,8,15,16$, and 17). The total PSQ score could be calculated as the mean of all items, excluding the three non-painful items (Appendix 1) [3,6].

\section{Translation}

The 'forward-backward' procedure was applied to translate the PSQ from English into Persian (Iranian language). Two individuals unconnected to the study translated the questionnaire into Persian. Both translators were instructed to aim for conceptual rather than literal translation [7]. The research team compared translations and produced a single provisional version of the questionnaire. Two other professional translators translated the provisional Persian questionnaire back into the English language [8]. Finally, an expert committee consisting of the translators, and the researchers reviewed the translation and the pre-final Persian version of the questionnaire was produced.

\section{Face validity}

A number of patients with low back pain completed the pre-final Persian version of the PSQ to establish that this version could be understood and that the questions measured what they were intended to measure. For each item patients were asked to respond to the following questions: "Do you understand what this means?" and "What does this mean to you by your own words?". Most patients correctly understood the questionnaire and the concept of each item. However, their general comments on difficulty in completing the questionnaire or understanding the texts were examined, and after a consensus by authors the final version was developed and used in this study.

\section{Patients and data collection}

The final draft of the Iranian version was administered to a sample of newly diagnosed LDH patients attending the neurosurgery clinic of a large teaching hospital in Tehran, Iran, between February 2011 and May 2013. There were no restrictions on patient selection with regard to types of $\mathrm{LDH}$, age or other characteristics. Patients who had lateral or central stenosis of spinal canal and previous surgery were excluded. The matched control group without LDH and other diseases were also selected. They were consisted of healthy cases that presented to the clinic for routine checkups. Cases in the control group had proved normal development in visits by physician. A skilled occupational therapist was collected the data for both groups and a skilled neurosurgeon performed the evaluation. 


\section{Additional measures}

The Iranian version of the Pain Catastrophizing Scale (PCS) was developed by Sullivan et al. [9] and has been widely used in the research of chronic pain and pain behavior in patients with low back pain [10]. This is a 13item instrument to measure the degree of catastrophizing. Each item evaluates the degree of patient's feelings or thoughts in painful situations or during pain anticipations. The scale includes three sub-scales: rumination, magnification and helplessness. The PCS total score could be computed by summing responses to all 13 items. Patients respond to each question according to their subjective mental preoccupation on a 5-point Likert Scale ranging from 0 (not at all) to 4 (all the time). The PCS total score ranges from 0 to $52[9,11]$. Lower scores on the PCS indicate better conditions. We used the total PCS score for this study.

The Finneson-Cooper score was also used. This is a lumbar disc surgery predictive score card or questionnaire that was developed to assess potential candidates for excision of a herniated lumbar disc [12]. The FinnesonCooper score ranges from 0 to 100 , and it categorizes candidates into a 4-grade classification: good $>75$; fair $65-75$; marginal 55-64, and poor $<55$.

\section{Statistical analysis}

Instruments measuring patient reported outcomes should satisfy certain psychometric properties including reliability and validity. Reliability refers to the degree to which an assessment tool or a questionnaire produces stable and consistent results. Validity is the extent to which an assessment tool or a questionnaire accurately measures what it purports to measure. Thus we performed the following analyses to assess psychometric properties of the PSQ.

\section{1) Reliability}

To test reliability the internal consistency of the questionnaire was measured using the Cronbach's alpha coefficient and alpha $\geq 0.70$ was considered satisfactory [13]. In addition, we performed an assessment of the test-retest reliability by comparing replies to the first and second administrations of the PSQ using the intraclass correlation coefficients (ICCs). All patients in LDH group were reexamined twice at 2 -week intervals. ICC $>0.80$ indicated excellent stability [13].
2) Validity

Validity was assessed performing item-scale correlations. Correlations were calculated using the Pearson's correlation coefficient $(r)$. It was expected that item scores would correlate higher with own hypothesized scale than other scales. Correlation values $\geq 0.40$ were considered satisfactory ( $r \geq 0.81-1.0$ as excellent, $0.61-0.80$ very good, $0.41-0.60$ good, $0.21-0.40$ fair, and 0.20 poor) [13]. In addition, the correlation between the PSQ and PCS was assessed using Pearson's correlation coefficient to assess criterion validity (convergent validity). Values $\geq 0.40$ were considered satisfactory $(r \geq 0.81-1.0$ as excellent, $0.61-0.80$ very good, $0.41-0.60$ good, $0.21-0.40$ fair, and $0.0-0.20$ poor) [13]. In addition, known-groups comparison analysis was performed to test how well the questionnaire discriminates between subgroups of patients who differed in the Finneson-Cooper score and between LDH group and normal cases. It was hypothesized that patients with a Finneson-Cooper "good" grade would achieve a greater increase in the PSQ score compared with cases with a Finneson-Cooper "fair" grade and the LDH group would report worse conditions on the PSQ compared to patients with normal cases.

\section{Ethics}

The Ethics Committee of Shahid Beheshti University of Medical Sciences approved the study.

\section{Results}

In all 140 patients completed the questionnaire. The characteristics of patients and their scores on the PSQ and affected levels are shown in Table 1. The mean age of patients was 51.7 (standard deviation $[\mathrm{SD}]=8.9$ ) years; most were married $(77.1 \%)$, and had completed primary or secondary education $(72.1 \%)$.

The internal consistency of the PSQ was found to be satisfactory reliability (Table 2). The Cronbach's alpha coefficient for the PSQ-minor, PSQ-moderate and PSQtotal was $0.80,0.82$ and 0.81 , respectively. Further analysis also indicated that the test-retest ICC (95\% confidence interval) was $0.84(0.616-0.932)$ for PSQ total, 0.85 (0.645-0.941) for PSQ-minor, and 0.84 (0.621-0.936) for PSQ-moderate for $\mathrm{LDH}$ group $(\mathrm{n}=89)$, lending support to its good reliability.

Validity of the PSQ was examined using item-scale cor- 
Table 1. The characteristics of the study patients and their scores on the PSQ $(n=140)$

\begin{tabular}{|c|c|c|c|}
\hline Characteristics & LDH group (n=101) & Control group ( $n=39)$ & $p$-value ${ }^{\text {a) }}$ \\
\hline Age & $52.4(9.1)$ & $49.9(8.7)$ & 0.319 \\
\hline Range & $31-64$ & $25-59$ & \\
\hline Sex & & & 0.776 \\
\hline Male (n, \%) & $47(46.5)$ & $15(38.5)$ & \\
\hline Female $(n, \%)$ & $54(53.5)$ & $24(61.5)$ & \\
\hline Educational status & & & 0.875 \\
\hline Illiterate & $17(16.8)$ & $4(10.2)$ & \\
\hline Primary & $46(45.5)$ & $12(30.8)$ & \\
\hline Secondary & $28(27.7)$ & $15(38.5)$ & \\
\hline College/university & $10(9.9)$ & 8 (20.5) & \\
\hline Marital status & & & 0.689 \\
\hline Single & $15(14.9)$ & $6(15.4)$ & \\
\hline Married & $79(78.2)$ & $29(74.4)$ & \\
\hline Divorced/widowed & $7(6.9)$ & $4(10.2)$ & \\
\hline Levels of LDH & & & - \\
\hline L3-4 & $8(7.9)$ & - & \\
\hline$\llcorner 4-5$ & $57(56.4)$ & - & \\
\hline L5-S1 & $36(35.6)$ & - & \\
\hline Finneson-Cooper score & & & - \\
\hline Good & $62(61.4)$ & - & \\
\hline Fair & $39(38.6)$ & - & \\
\hline \multicolumn{4}{|l|}{$\mathrm{PCS}^{\text {bl }}$} \\
\hline PCS (0-52) & $26.1(10.1)$ & $13.1(9.2)$ & $<0.001$ \\
\hline \multicolumn{4}{|l|}{ PSQ $^{c l}$} \\
\hline PSQ-minor (0-10) & $5.3(2.0)$ & $2.1(1.0)$ & $<0.001$ \\
\hline PSQ-moderate (0-10) & $6.4(1.8)$ & $3.9(1.1)$ & $<0.001$ \\
\hline PSQ-total (0-10) & $5.9(1.9)$ & $3.1(1.1)$ & $<0.001$ \\
\hline
\end{tabular}

Values are presented as mean (standard deviation).

PSQ, Pain Sensitivity Questionnaire; LDH, disc herniation.

a) Statistical significance of difference between groups is tested by chi-square $\left(\chi^{2}\right)$ test for categorical data and by Mann-Whitney $U$ test for con-

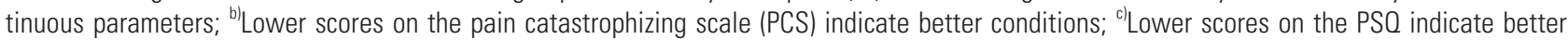
conditions.

Table 2. Test-retest reliability results for the PSQ-minor, PSQ-moderate and PSQ-total scores for LDH group (n=89)

\begin{tabular}{lcccc} 
PSQ-measure & Mean (SD) & No. of items & Cronbach's alpha & ICC $^{\text {b) }}$ \\
PSQ-minor & $5.3(2.0)$ & 7 & 0.80 & 0.85 \\
PSQ-moderate & $6.4(1.8)$ & 7 & 0.82 & 0.84 \\
\hline PSQ-total & $5.9(1.9)$ & 14 & 0.81 & 0.84 \\
\hline
\end{tabular}

PSQ, Pain Sensitivity Questionnaire; LDH, lumbar disc herniation; SD, standard deviation; ICC, intraclass correlation coefficient.

${ }^{a)} A$ value of 0.70 or above indicates adequate reliability; ${ }^{b} A$ value of ICC above 0.80 was considered as evidence of excellent reliability.

relations. The item-scale correlation matrix between each item and the two PSQ subscales based on LDH disease are shown in Table 3. All correlations between items and its hypothesized scale showed satisfactory results suggest- 
Table 3. Item-scale correlation matrix for the two Pain Sensitivity Questionnaire (PSQ) subscales ${ }^{\text {a) }}(\mathrm{n}=140)$

\begin{tabular}{|c|c|c|}
\hline Items (item number) & PSQ-minor & PSQ-moderate \\
\hline $\begin{array}{l}\text { 1. Imagine you bump your shin badly on a hard edge, for example, on the edge of a glass } \\
\text { coffee table }\end{array}$ & 0.15 & 0.68 \\
\hline 2. Imagine you burn your tongue on a very hot drink & 0.27 & 0.71 \\
\hline 3. Imagine your muscles are slightly sore as the result of physical activity & 0.82 & 0.17 \\
\hline 4. Imagine you trap your finger in a drawer & 0.22 & 0.74 \\
\hline 5. Imagine you take a shower with lukewarm water. & 0.22 & 0.21 \\
\hline 6. Imagine you have mild sunburn on your shoulders. & 0.69 & 0.22 \\
\hline 7. Imagine you grazed your knee falling off your bicycle & 0.75 & 0.16 \\
\hline 8. Imagine you accidentally bite your tongue or cheek badly while eating & - & 0.69 \\
\hline 9. Imagine walking across a cool tiled floor with bare feet. & 0.19 & 0.23 \\
\hline 10. Imagine you have a minor cut on your finger and inadvertently get lemon juice in the wound & 0.51 & 0.19 \\
\hline 11. Imagine you prick your fingertip on the thorn of a rose. & 0.71 & 0.24 \\
\hline $\begin{array}{l}\text { 12. Imagine you stick your bare hands in the snow for a couple of minutes or bring your hands } \\
\text { in contact with snow for some time, for example, while making snowballs }\end{array}$ & 0.76 & 0.25 \\
\hline 13. Imagine you shake hands with someone who has a normal grip. & 0.14 & 0.19 \\
\hline 14. Imagine you shake hands with someone who has a very strong grip & 0.65 & 0.23 \\
\hline 15. Imagine you pick up a hot pot by inadvertently grabbing its equally hot handles & 0.14 & 0.51 \\
\hline 16. Imagine you are wearing sandals and someone with heavy boots steps on your foot & 0.17 & 0.71 \\
\hline 17. Imagine you bump your elbow on the edge of a table ("funny bone") & 0.23 & 0.76 \\
\hline
\end{tabular}

Bold text indicates a statistically significant.

${ }^{\text {al }}$ Pearson correlation $(r)$ equal to or greater than 0.40 was considered satisfactory (correlation $\geq 0.81-1.0$ as excellent, $0.61-0.80$ very good, $0.41-0.60$ good, $0.21-0.40$ fair, and $0.0-0.20$ poor) [13].

ing that the items had a substantial association with the subscale representing the concept. Pearson correlation coefficient exceeded the 0.40 level recommended ranging from $0.51(\mathrm{Q} 10)$ to $0.82(\mathrm{Q} 4)$. In addition, the PSQ demonstrated a significant positive correlation with PCS lending support to its good convergent validity $(r=0.81$; $p<0.001$ ) for patients with $\mathrm{LDH}$.

Validity of the PSQ was examined using the known groups comparison. The PSQ scores well discriminated between LDH group and normal cases $(p<0.001)$. In addition, the patients' rating on the PSQ assessments discriminated well between sub-groups of patients who differed in the Finneson-Cooper score. The total PSQ score by Finneson-Cooper score for Good (Finneson-Cooper score $>75$ ) and Fair (Finneson-Cooper score 65-75) were $7.1(\mathrm{SD}=1.8)$ and $4.1(\mathrm{SD}=1.9)$, respectively. The PSQ score was significantly higher for those who were identified as "good" by the Finneson-Cooper score, as hypothesized $(p<0.001)$. Additionally, the total PSQ was also significantly correlated with the total scores of the PCS, lending support to its good convergent validity.

\section{Discussion}

This study is the first to report on translation and validation of the PSQ in Iran. The results of the current study showed that the Persian version of the PSQ is a reliable measure to evaluate pain in Iranian patients with LDH. The Cronbach alpha for the Iranian version of PSQ exceeded the recommended threshold, suggesting that the Persian version of the questionnaire has satisfactory internal consistency. The results are similar to those reported by other authors who have used this measure in patients with degenerative spinal disease and chronic pain $[3,5]$.

Test-retest reliability was examined using ICCs. All ICCs of the subscales were $>0.80$, demonstrating good test-retest reliability for the scale, similar to the original version of PSQ [3]. However, it was higher compared to other studies due to variety of intervals $[3,14]$.

Significant correlations were also found between the 
PSQ and the previously validated PCS. These results match the good construct validity reported in similar studies made in other language [5] and original version of PSQ [3]. Sellers et al. [4] was also observed similar results between the PSQ and visual analog scale. In addition, item-scale correlation for two subscales of the PSQ (PSQminor and PSQ-moderate) was calculated a satisfactory result was observed. Item-scale correlation was not assessed in other studies.

The known-groups comparison demonstrated that the PSQ was able to significantly discriminate between healthy cases and patients with LDH. Ruscheweyh et al. [14] were also observed similar results between patients with chronic pain and healthy controls. In addition, we used the Finneson-Cooper score as a clinical measure for known-groups comparison. The findings showed that patients who differed in Finneson-Cooper score assessments scored differently on the PSQ score, as expected. In fact such a result lends support to the discriminant validity of the PSQ score.

Interestingly, we found that the PSQ score was higher in the group with "good" Finneson-Cooper score compared to those who identified as "fair". In fact, PSQ was able to differentiate between those who were good candidates for surgery compared to those who were not, indicating that the outcome of surgery might not be satisfactory for the group with fair Finneson-Cooper score.

Although, psychometric evaluation were different in many studies, as in the Korean (5), German (3), English (4), psychometric studies, the results of our studies indicated similarly good, construct validity, and internal consistency. To the authors' best knowledge, the Persian version of the PSQ is the only condition-specific pain sensitivity measures for patients with $\mathrm{LDH}$ that were undergone psychometric evaluation in Iran. The results of the current study show that, this instrument seems to be reliable and valid outcome measure for evaluating of patients with LDH in Iran. We encourage professionals to continue to study the test using different samples, statistical procedures, other disease, and related measure to clarify its application in practice.

We carried out a number of limited tests to perform this validation study. In the future, it might be necessary to perform other statistical tests to establish stronger psychometric properties for the PSQ score. Potential research should focus on performing both explanatory factor analysis and confirmatory factor analysis to insure its psychometrics properties. Future research with the PSQ in larger populations of patients with the variety of $\mathrm{LDH}$ is required to further test the factor structure of the PSQ as a population health measure in Iran. Nevertheless, our main recommendation is to use simple, economical alternative and is a quick and effective alternative in clinical practice to assess pain sensitivity.

\section{Conclusions}

This study showed that the adapted Iranian PSQ is a valid and reliable questionnaire for the assessment of pain in patients with $\mathrm{LDH}$.

\section{Conflict of Interest}

No potential conflict of interest relevant to this article was reported..

\section{Acknowledgments}

The authors thank the staff of the Neurosurgery Unit at Imam-Hossain Hospital, Tehran, Iran.

\section{References}

1. Fardon DF, Milette PC; Combined Task Forces of the North American Spine Society, American Society of Spine Radiology, and American Society of Neuroradiology. Nomenclature and classification of lumbar disc pathology. Recommendations of the Combined task Forces of the North American Spine Society, American Society of Spine Radiology, and American Society of Neuroradiology. Spine (Phila Pa 1976) 2001;26:E93-113.

2. Nielsen CS, Staud R, Price DD. Individual differences in pain sensitivity: measurement, causation, and consequences. J Pain 2009;10:231-7.

3. Ruscheweyh R, Marziniak M, Stumpenhorst F, Reinholz J, Knecht S. Pain sensitivity can be assessed by self-rating: Development and validation of the Pain Sensitivity Questionnaire. Pain 2009;146:65-74.

4. Sellers AB, Ruscheweyh R, Kelley BJ, Ness TJ, Vetter TR. Validation of the English language pain sensitivity questionnaire. Reg Anesth Pain Med 2013;38:50814.

5. Kim HJ, Ruscheweyh R, Yeo JH, et al. Translation, 
cross-cultural adaptation, and validity of the Korean version of the pain sensitivity questionnaire in chronic pain patients. Pain Pract 2014;14:745-51.

6. Kim HJ, Lee JI, Kang KT, et al. Influence of pain sensitivity on surgical outcomes after lumbar spine surgery in patients with lumbar spinal stenosis. Spine (Phila Pa 1976) 2015;40:193-200.

7. World Health Organization. Process of translation and adaptation of instruments [Internet]. Geneva: World Health Organization; 2016 [cited 2016 May 2]. Available from: http://www.who.int/substance_ abuse/research_tools/translation/en/.

8. Bullinger M, Alonso J, Apolone G, et al. Translating health status questionnaires and evaluating their quality: the IQOLA Project approach. International Quality of Life Assessment. J Clin Epidemiol 1998; 51:913-23.

9. Sullivan MJL, Bishop SR, Pivik J. The pain catastro- phizing scale: development and validation. Psychol Assess 1995;7:524-32.

10. George SZ, Calley D, Valencia C, Beneciuk JM. Clinical Investigation of Pain-related Fear and Pain Catastrophizing for Patients With Low Back Pain. Clin J Pain 2011;27:108-15.

11. Raeissadat SA, Sadeghi S, Montazeri A. Validation of the pain catastrophizing scale (PCS) in Iran. J Basic Appl Sci Res 2013;3:376-80.

12. Finneson BE, Cooper VR. A lumbar disc surgery predictive score card: a retrospective evaluation. Spine (Phila Pa 1976) 1979;4:141-4.

13. Nunnally JC, Bernstein IH. Psychometric theory. New York: McGraw-Hill; 1994.

14. Ruscheweyh R, Verneuer B, Dany K, et al. Validation of the pain sensitivity questionnaire in chronic pain patients. Pain 2012;153:1210-8. 
Appendix 1. Pain Sensitivity Questionnaire

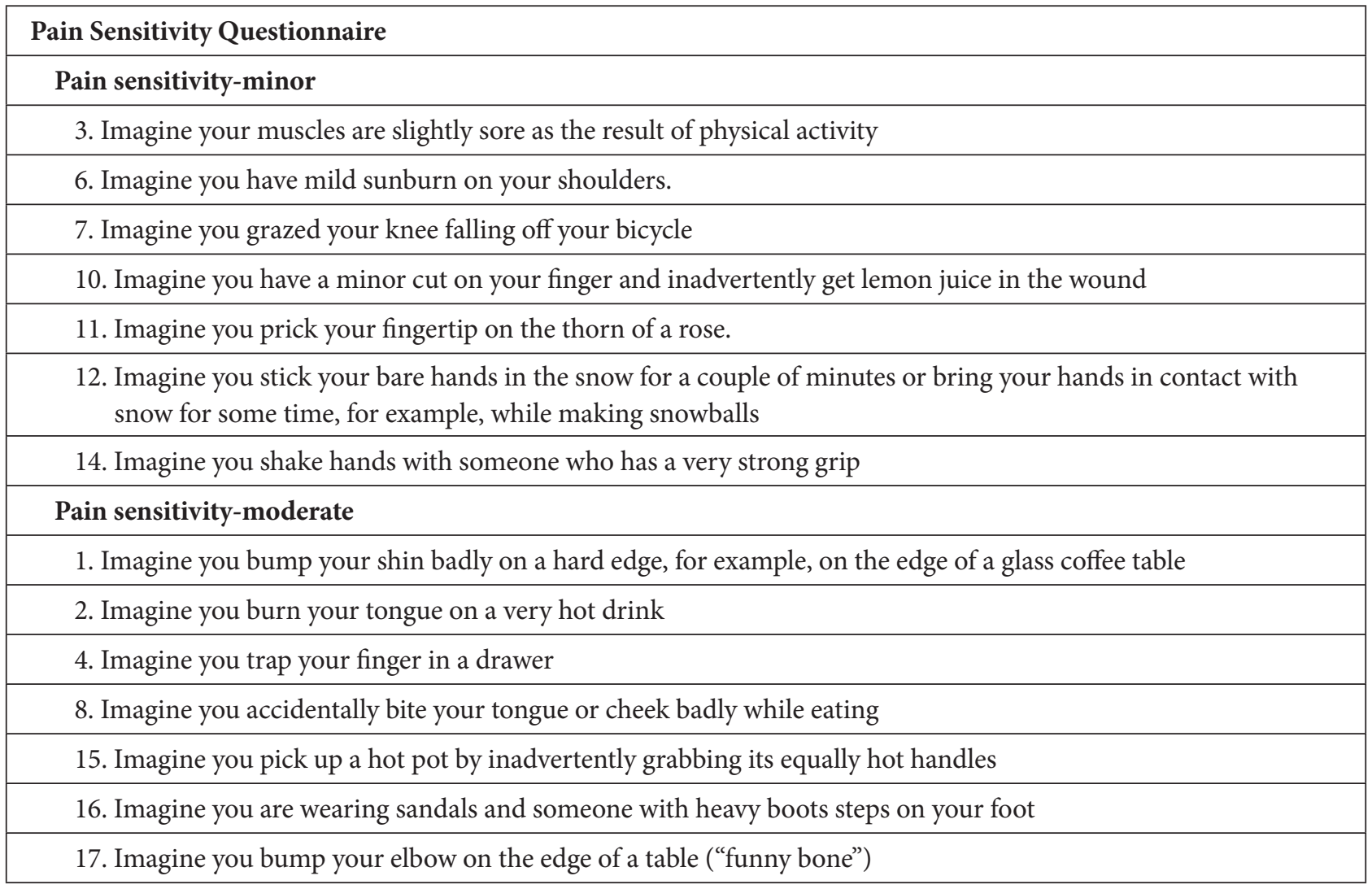

